\title{
Feasibility of a magma ocean dynamo on Mars
}

\author{
George Helffrich (1)
}

\begin{abstract}
Crustal magnetization of rocks in regions of Mars surface testifies to an era of dynamo activity. I examine the possibility that the dynamo that operated then, in the first 400-600 Ma after Mars formed, was powered by a crystallizing subsurface magma ocean. Of the ways that a magma ocean dynamo could operate, on Mars only turbulent and magnetostrophic dynamos seem feasible; geostrophic dynamos do not seem so unless very high heat flows, 100-1000 times present, are invoked. Given the anticipated information from the future InSight lander mission, it will be difficult to assess where the dynamo originated unless an inner core is discovered, rendering the dynamo likely to have operated in the core.
\end{abstract}

Keywords: Mars, Dynamo, InSight, Magma ocean

\section{Introduction}

Mars' era of dynamo activity was discovered when the Mars Global Surveyor (MGS) spacecraft dropped to a low orbit during an aerobraking manoeuver and recorded quite high levels of crustal magnetization (Acuña et al. 1999). The heavily cratered, older southern highlands exhibit the strongest signals, restricted to the crust; there is no presently operating dynamo due to the lack of a planetary field detectable from high orbit. The much lower levels of crustal magnetism in the younger, northern planetary hemisphere (100 times smaller according to Whaler and Purucker (2005)), led the discoverers to infer that a dynamo that operated early in Mars' history up until the early Noachian epoch (for $\sim 0.5$ Gyr). Some debate on the validity of this inference ensued until the magnetization of individual crystals in SNC meteorites was measured and the same crystals were dated, showing magnetic ages of 3.9-4.1Ga (Weiss et al. 2002). More recent estimates based on crater counts overlap with the age range $4.1 \pm 0.2 \mathrm{Ga}$ (Chassefière et al. 2007) further supporting this conclusion.

Dynamos require a power source to operate. They are driven by fluid motions in conductive materials, commonly assumed to be the planet's core. If there is no motion, the field will decay on a time scale given by

Correspondence: george@elsi.jp

Earth-Life Science Institute, Tokyo Institute of Technology, 2-12-1-IE-1

Ookayama, Meguro-ku, Tokyo 152-8550, Japan the magnetic diffusivity. For the Earth, this decay time is around 10,000 years (Stevenson 2003) and, given Mars' smaller core size, corresponding shorter by the square of the radius ratio, or 2500 years.

There are three viable sources for powering dynamos. The first is heat from cooling of the core or a thermal dynamo. This may be accretion heat (essentially released gravitational potential energy) or radiogenic heating of the core itself. Most radiogenic elements with long lifetimes (U, Th, K) are lithophile (McDonough and Sun 1995) and do not significantly concentrate themselves in the core. Thus radioactivity is probably not a significant factor in powering thermal dynamos.

The second is by crystallization of a solid inner core out of the liquid outer core, a significant contribution to the power for the Earth's dynamo now (Labrosse 2003). The crystallization not only releases latent heat, it also enriches the alloying elements in the core liquid by excluding them from the solid. The exclusion makes the remaining core liquid less dense and thus causes it to rise buoyantly from the crystallizing region, essentially recovering gravitational potential energy. This fluid motion is able to drive the dynamo quite efficiently and for a long time. For example, scaling laws for the growth of the Earth's inner core (Breuer et al. 2010) suggest that it requires another 10-20 Gyr to crystallize the whole core and thus extinguish dynamo activity. This dynamo may be 
described as a compositional type because it is the changing composition of the crystallizing fluid that primarily contributes to its running.

The final way to drive a dynamo is by precession of the solid body surrounding the core forced by tidal coupling to the planet's rotational bulge. Though this seems energetically efficient, conditions for its operation have not been explored as thoroughly as the other two (Wu and Roberts 2009). In the case of Mars, with no present-day field, and yet tidal interactions with its moons Diemos and Phobos, it clearly does not operate now and, by inference, probably never did.

Now, consider what type of dynamo Mars' early one might have been. If it was compositional, then it should still be running now, or Mars' core must be solid. The latter possibility is ruled out by the elastic tidal Love number $k_{2}$, whose value is $0.159 \pm 0.009$, which indicates that the core is at least partly liquid (Konopliv et al. 2006). It is possible that the core is not completely frozen, and the annulus of remaining liquid is too thin to support dynamo activity (Stevenson 2001). This state must have been achieved by $\sim 500 \mathrm{Myr}$, which is about the limit age of magnetized areas of the Martian crust. Again, using the scaling laws for inner core growth (Breuer et al. 2010), the growth time requires that the inner core nucleated quite early in Mars' accretion history; 100 year after core formation. Given that accretion times are on the order of 10 Myr (Brasser 2013), the scaling places the inner core's (and thus its magnetic field's) onset time before accretion's end and requires inner core growth during accretion and during growth of the core itself. If accretion is violently cataclysmic, physical disruption of the core renders this scenario implausible.

It is also possible that compositional dynamos might be run by crystallization elsewhere in the core rather than its center. Breuer et al. (2010), summarizing earlier suggestions (Stevenson 1983), note that saturation of metal in light elements might occur at the lower pressures of a magma ocean that might later exsolve from the metal due to oversaturation at higher pressures after the metal segregates to form the core. Flow of exsolved material upwards in the core could drive a dynamo, which Hirose et al. (2017) show that it is quite efficient and possibly operates the present-day Earth's. No computationally simulated dynamos have yet been constructed to operate in this regime, though Hori et al. (2012) note that volumetric buoyancy sources, which could characterize exsolutiondriven dynamos, yield more multipolar fields and hence less spatial regularity to the field, perhaps mimicking Mars' surface magnetism. If, however, the Earth's dynamo operates this way (Hirose et al. 2017), its strong dipolarity contradicts the volumetric source field morphology inference.
Consequently, thermal dynamos seem apposite for Mars. Thermal dynamos have a characteristic property that if conditions for running them become unfavorable anywhere in a quasi-adiabatic, convecting core, they are unfavorable everywhere in the quasi-adiabatic region (Stevenson 2001). Thus, thermal dynamos essentially are either on or off, controlled by the flux of heating down the adiabatic gradient to the surface of the core from its interior. This provides a way to end dynamo activity simply by regulating the heat drawn from the core into the mantle. If Mars had an early plate tectonic era (Anguita et al. 2006) that evolved into a stagnant lid form of convection (O'Neill et al. 2007), heat flowing out of the core would wane with the change in tectonic style.

A few Mars development scenarios are based on this phenomenon. Williams and Nimmo (2004) suggest that Mars' core formed quite rapidly and was $\sim 150 \mathrm{~K}$ hotter than the base of the mantle. With that extra heat, a dynamo could operate by simple core cooling by convective withdrawal of heat by the mantle from the coremantle boundary, with the mantle operating in a stagnant lid regime (i.e., no plate tectonics). After $\sim 0.5 \mathrm{Gyr}$, the core would be cooled sufficiently that dynamo action would stop. Even added radiogenic ${ }^{40} \mathrm{~K}$ in the core is not able to provide enough extra heat early in Mars' history to run a dynamo. In their view, Mars' core is still entirely liquid.

Reese and Solomatov (2010) invoke a giant impact origin for the Martian dynamo. The impact causes superheating of Mars' core and thus is a more elaborate form of the previous model. They simulated impact of a planetesimal with Mars and the transfer of the impact-derived heat of the differentiated impactor's metal to the previously formed core, stratifying the hotter metal onto the core. This model attributes the southern highland crust to new crust formed after recrystallization after the impact, inverting the usual relative age relations obtained from crater counts (Frey 2006a, b). One further insight that this study provides is that the core may develop in a state where its exterior is hotter than its interior due to the heat gained by the accumulated metal at the base of a magma ocean descending in the gravitational field of the planet. This temperature profile in the core suppresses convection and thus any dynamo activity. Hence, one also needs to examine whether dynamo activity is possible in the mantle while it is still wholly or partly liquid in the magma ocean phase of development, one of the motivations for this work.

Elkins-Tanton et al. (2005) use the evolution of convection in the early Mars mantle to cause its core to be thermally perturbed into convective motion. The progressive crystallization of an early magma ocean led to the enrichment of denser, iron-rich material at its top in its final stages. This is a convectively unstable configuration 
that eventually overturns, bringing cold material into contact with the core and starting convection in it due to high heat flow across the core-mantle boundary. The convection is transient, lasting 15-150 Myr, ending because the mantle warms and evolves to an adiabatic state that no longer draws sufficient heat from the core to cool it and run its dynamo.

A final intriguing possibility is that the core was not involved in early dynamo activity on Mars at all. Rather, the dynamo was in the mantle as it cooled through the magma ocean phase after having developed an early crust (Stevenson 2001). Stegman et al. (2003) investigated this possibility for running a lunar dynamo, and Ziegler and Stegman (2013) similarly sought an explanation for Earth's early magnetic field by this mechanism. Here, we apply the same methods to investigate whether a mantle dynamo is feasible for Mars.

\section{Planetary dynamo styles}

Dynamos arise from the interplay of convection in a conductive material and electromotive forces arising from the convective motions that generally act to resist motion. They can be characterized by the dominant balance of forces that maintain steady state convection-steady in the sense that though the motion may reorganize itself and adopt different flow patterns, it does not stop. From them, governing relations for characteristic convective speeds may be obtained (Christensen 2010). A brief introduction to the styles arising in magma ocean dynamos follows.

In the style of magma ocean envisaged by Solomatov (2007), temperatures are high, viscosities low, and motion rapid. This leads to a highly turbulent flow regime which is indifferent to the planet's rotational state. Under these conditions, the buoyancy forces due to rising plumes of hot material and sinking plumes of cold material of possibly different composition (an additional source of buoyancy) are resisted by inertia of the fluid itself: packets of fluid need to move out of the way to allow the buoyant bits to flow (Starchenko and Jones 2002). In contrast, Starchenko and Jones (2002) think this force balance to be unrealistic given that rapid flows lead to correspondingly large magnetic forces that resist the flow and create electrical currents that dissipate energy through the electrical resistance of the fluid. They infer that the Lorenz force (the combination of electrical attraction/repulsion and an electrical charge's interaction with a magnetic field) must play a leading role in resisting motion. Moreover, the effects of rotation (giving rise to the Coriolis force) and pressure buildup in a fluid due to its being influenced by the Coriolis force also serve to counteract buoyancy-driven fluid movement. This leads to what they call a MAC force balance (Magnetic-Archimedian (buoyancy)-Coriolis).
A third convective style was motivated from observations of the patterns of convection in a rotating spherical container conducted by Aubert et al. (2001). They found that two main factors cooperated to balance the buoyancy forces: the fluid's inertia and the Coriolis force. This leads to a flow pattern organized into rotating columns that run parallel to the rotation axis through the height of the container. They call this a CIA force balance (Coriolis-InertiaArchimedian (buoyancy)). In contrast to the previous style, here, the Lorenz force does not play a role.

Which of these convective styles characterizes an early Mars magma ocean? It is not at all clear. Hence the implications of each will be assessed, and their likelihood for driving an early Mars dynamo will be evaluated.

\section{Methods/Experimental}

The controlling factor for a dynamo is its magnetic Reynolds number, $R_{m}$. It is defined through

$$
R_{m}=\nu L \mu_{0} \sigma
$$

where $\mu_{0}$ is a physical constant, $\sigma$ is electrical conductivity, $L$ is the length scale of the fluid flow (the mantle depth), and $v$ is a characteristic fluid velocity. We need $R_{m}$ to be greater than $\sim 40$ for dynamo activity to ensue (Olson and Christensen 2006). Silicate melt conductivity data is somewhat sparse, so I use 1 atm values for basaltic melt from Pommier et al. (2010) evaluated at a temperature of $1700 \mathrm{~K}$, which, according to Sohl and Spohn (1997), is a characteristic temperature in Mars' mid-mantle. This indicates a value for $\sigma$ of $8.9-22.9 \mathrm{~S} \mathrm{~m}^{-1}$. A characteristic speed is harder to specify because it depends on the governing process. The three processes identified by Ziegler and Stegman (2013) operating in rapidly rotating fluid systems are turbulence, the buoyancy-Lorenz force (magnetostrophic) balance, and the buoyancy-inertiacoriolis (geostrophic) force balance. These are expressed as follows:

$$
\begin{aligned}
v_{T} & =14\left[\frac{Q_{m} \alpha g}{\rho C_{\mathrm{P}}} \frac{1}{\Omega}\right]^{1 / 2} & \text { turbulence } \\
v_{\mathrm{MAC}} & =\left[\frac{Q_{m} \alpha g}{\rho C_{\mathrm{P}}} \frac{1}{\Omega}\right]^{1 / 2} & \text { buoyancy }- \text { Lorenz } \\
v_{\mathrm{CIA}} & =\left[\frac{Q_{m} \alpha g}{\rho C_{\mathrm{P}}}\right]^{2 / 5}\left[\frac{L}{\Omega}\right]^{1 / 5} & \text { buoyancy }- \text { inertia }- \text { coriolis }
\end{aligned}
$$

These in turn depend on $Q_{m}$, the heat flux through the convective region, the rotation rate $\Omega(2 \pi /$ day length in seconds), and other properties listed in Table 1. I evaluate $R_{m}$ for the various possibilities using Eqs. (1) and (2). 
Table 1 Thermophysical properties for Mars mantle relevant to dynamo activity

\begin{tabular}{llll}
\hline Property & Symbol & Value & Scale and dimensions \\
\hline Magnetic permeability & $\mu_{0}$ & $4 \pi$ & $\times 10^{-7} \mathrm{H} \mathrm{m}^{-1}$ (definition) \\
Thermal expansivity & $\alpha$ & 2.3 & $\times 10^{-5} \mathrm{~K}^{-1 \mathrm{a}}$ \\
Heat capacity & $C_{\mathrm{p}}$ & 1200 & $\mathrm{~J} \mathrm{~kg}^{-1} \mathrm{~K}^{-1 \mathrm{~b}}$ \\
Gravity & $g$ & 3 & $\mathrm{~m} \mathrm{~s}^{-2 \mathrm{a}}$ \\
Density & $\rho$ & 4000 & $\mathrm{~kg} \mathrm{~m}^{-3 \mathrm{a}}$ \\
Rotation rate & $\Omega$ & 71 & $\times 10^{-6} \mathrm{~s}^{-1 \mathrm{c}}$ \\
Heat flux & $Q_{m}$ & 519 & $\mathrm{GW}, \mathrm{c}$ \\
\hline
\end{tabular}

a Sohl and Spohn (1997) evaluated in the transition zone

b Nimmo and Stevenson (2000)

c Present day value

\section{Results and Discussion}

The sensitivity to these parameters is shown in Fig. 1. It shows that turbulent flow speeds $\left(v_{T}\right)$ and magnetostrophic flow speeds $\left(v_{\text {MAC }}\right)$ are capable of operating a dynamo in Mars' mantle $\left(R_{m}>40\right)$, provided that it is liquid. It appears more difficult to do this with geostrophic flows $\left(v_{\mathrm{CIA}}\right)$ because the required heat flows are too large: 100-1000 times the present-day estimates. Convection models for the evolution of heat flow of Mars (Breuer et al. 2010) show that heat flows high enough to run a magnetostrophic mantle dynamo existed between 0-1 Gyr after accretion ended, which would provide the required heat flow increase in the time frame for the dynamo's existence. It therefore seems possible that the earliest-surviving crust in the magma ocean phase of planetary evolution could record a magnetic field generated by a mantle dynamo, independent of whether a core dynamo was also operating. As the magma ocean internally crystallized, its depth $L$ lessens, eventually dropping $R_{m}$ below the critical value (Eq. (1)). Thus, the last formed magmatic output would be emplaced without any internal field.

Takahashi and Matsushima (2006) showed that some thin annular dynamos are non-dipolar, in contrast to thicker shelled dynamos that, except where driven by internal heat generation or are at high Rossby number $R o=v(\Omega L)^{-1}$, are dipolar (Olson and Christensen 2006). The magma ocean dynamos considered here have $R o \leq 1 \times 10^{-5}$, decidedly low, so their behavior will be controlled by their annular depth and will be nondipolar. Consequently, as the magma ocean crystallizes, any dynamo operating in it will become weaker and multipolar. This may account for the lack of coherently magnetized domains in the Martian highlands Connernery et al. (1999). The Martian dynamo may have been reversing, an inference from the initial discovery of the pattern of crustal magnetization (Connerney et al. 1999) that showed stripes of alternately polarized magnetic domains evocative of seafloor magnetism on the Earth. This concept involves crustal age variations recording epochs of different polarity, whose analogous mechanism on Mars is not obvious. Rather, later interpretations invoke nonspecific mechanisms involving crustal recycling to explain the polarity variation (Whaler and Purucker 2005), yet they still assume age differences for differently magnetized areas of the crust. Cratering records seem to bear this out (Chassefière et al. 2007), as they do for the weak-to-absent crustal magnetization of the Northern hemispheric dichotomy and the Argyre and Hellas impact basins (Acuña et al. 1999; Whaler and Purucker 2005).

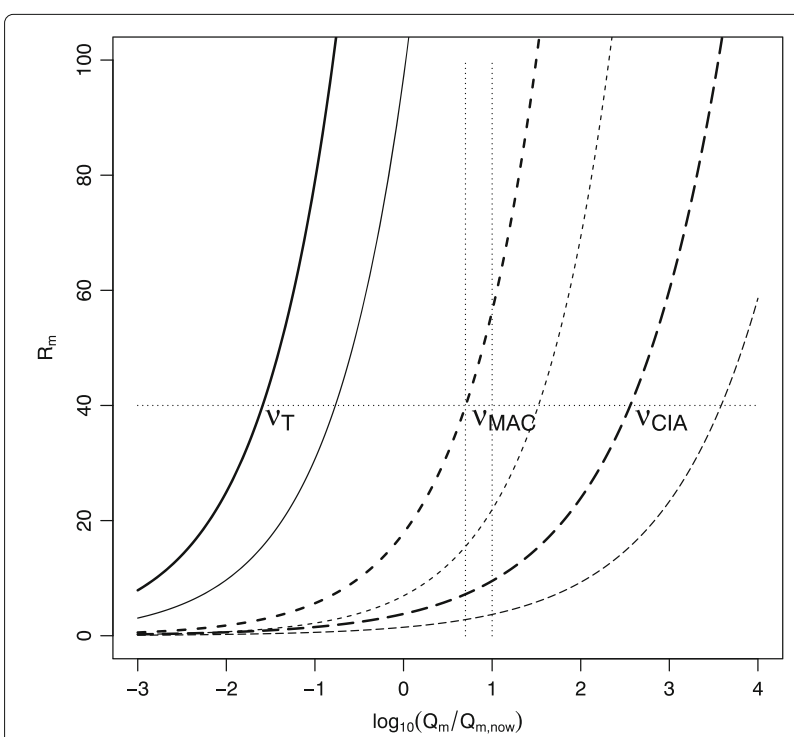

Fig. 1 Feasibility of running a dynamo in a liquid silicate mantle in Mars as a function of mid-mantle heat flux $Q_{m}$. The heat flux is scaled relative to the present-day heat flux $Q_{m \text {,now }}$ as estimated by Sohl and Spohn (1997). $v_{T}, v_{M A C}$, and $v_{C I A}$ show three families of potential dynamos driven by different types of flows (see text). Thick and thin lines of each pair denote low and high estimates of silicate electrical conductivity, $\sigma\left(8.9-22.9 \mathrm{~S} \mathrm{~m}^{-1}\right)$. $R_{m}$ values greater than 40 (horizontal dotted line) indicate feasible range for dipolar dynamos. Turbulent flow speeds are capable of running dynamos at any mantle heat flow provided that the mantle is liquid. Magnetostropic dynamos are also feasible in the high heat flux epoch early in Mars evolution (vertical dotted lines; 0-1 Gyr according to Breuer et al. (2010)). Geostrophic dynamos do not seem feasible under any realistic heat flow scenario 
Nimmo (2000) proposes that the origin of the magnetic lineations is dike emplacement in the crust, a process that requires crustal extension and moving intrusion loci in space and time. No matter what the dynamo origin, the crustal magnetization pattern apparently necessitates contemporaneous tectonism, which the geological evidence elsewhere supports (Anguita et al. 2006).

\section{Conclusions}

In the near future, after the InSight lander mission (NASA 2013) places a seismometer on Mars and records sufficient seismic activity around and within the planet (Taylor et al. 2013), it should be feasible to use the resulting data to draw stronger inferences about Mars' composition and evolution. First-order constraints on the planetary $\mathrm{Fe} / \mathrm{Si}$ ratio (Rivoldini et al. 2011; Sohl and Spohn 1997) will be provided by the radius of Mars' core, which will be evident from core-reflected P and S (PcP and ScS) and core penetrating (SKS, SKKS) travel times. The next most important seismic observation relevant for dynamo activity will be the presence or absence of an inner core. If an inner core is detected, the dynamo had a compositional power source available and the straightforward inference is to assume that the dynamo originated in the core. Absence of an inner core will still not discriminate between a mantle and a core dynamo, nor whether any core dynamo was thermal or compositional, unless there is some seismic evidence of reaction between an exsolved core component and the mantle or in the core's wave speed structure itself.

\section{Acknowledgements \\ I thank John Hernlund and Hiroaki Matsui for discussions on the subject of dynamos and James Dohm for insights into Martian geology.}

\section{Funding}

This work was not funded by any agency whatsoever; the work arose from the circumstances of day-to-day activities at my home institute.

\section{Authors' contributions}

All authors read and approved the final manuscript.

\section{Competing interests}

The author declares that he has no competing interests.

\section{Publisher's Note}

Springer Nature remains neutral with regard to jurisdictional claims in published maps and institutional affiliations.

Received: 9 January 2017 Accepted: 6 July 2017

Published online: 15 August 2017

\section{References}

Acuña MH, Connerney JEP, Ness NF, Lin RP, Mitchell D, Carlson CW, McFadden J, Anderson KA, Rème H, Mazelle C, Vignes D, Wasiliewski P, Cloutier P (1999) Global distribution of crustal magnetization discovered by the Mars Global Surveyor MAG/ER experiment. Science 284:790-793

Anguita F, Fernandez C, Cordero G, Carrasquilla S, Anguita J, Nunez A, Rodriguez S, Garcia J (2006) Evidences for a Noachian-Hesperian orogeny in Mars. Icarus 185:331-357

Aubert J, Brito D, Nataf H-C, Cardin P, Masson J-P (2001) A systematic experimental study of rapidly rotating spherical convection in water and liquid gallium. Phys Earth Planet Inter 128:51-74
Brasser R (2013) The formation of Mars: building blocks and accretion time scale. Space Sci Rev 174:11-25

Breuer D, Labrosse S, Spohn T (2010) Thermal evolution and magnetic field generation in terrestrial planets and satellites. Space Sci Rev 152:449-500

Chassefière E, Leblanc F, Langlais B (2007) The combined effects of escape and magnetic field histories at Mars. Planet Space Sci 55:343-357

Christensen UR (2010) Dynamo scaling laws and applications to the planets. Space Sci Rev 152:565-590

Connerney JEP, Acuña MH, Wasiliewski P, Ness NF, Rème H, Mazelle C, Vignes D, Lin RP, Mitchell D, Cloutier P (1999) Magnetic lineations in the ancient crust of Mars. Science 284:794-798

Elkins-Tanton LT, Zaranek SE, Parmentier EM, Hess PC (2005) Early magnetic field and magmatic activity on Mars from magma ocean cumulate overturn. Earth Planet Sci Lett 236:1-12

Frey HV (2006a) Impact constraints on, and a chronology for, major events in early Mars history. J Geophys Res 111:10-10292005002449

Frey HV (2006b) Impact constraints on the age and origin of the lowlands of Mars. Geophys Res Lett 33:10-10292005024484

Hirose K, Morard G, Sinmyo R, Umemoto K, Hernlund J, Helffrich G, Labrosse S (2017) $\mathrm{SiO}_{2}$ crystallization and compositional evolution of the Earth's core. Nature 543:99-102

Hori K, Wicht J, Christensen UR (2012) The influence of thermo-compositional boundary conditions on convection and dynamos in a rotating spherical shell. Phys Earth Planet Inter 196:32-48

Konopliv AS, Yoder CF, Standish EM, Yuan D-N, Sjogren WL (2006) A global solution for the Mars static and seasonal gravity, Mars orientation, Phobos and Deimos masses, and Mars ephemeris. Icarus 182:23-50

Labrosse S (2003) Thermal and magnetic evolution of the Earth's core. Phys Earth Planet Inter 140:127-143

McDonough WF, Sun S-s (1995) The composition of the Earth. Chem Geol 120:223-253

NASA NASA (2013) NASAfacts - InSight. (Publ. no. JPL 400-1513, Rev. 26/13)

Nimmo F (2000) Dike intrusion as a possible cause of linear Martian magnetic anomalies. Geology 28:391-394

Nimmo F, Stevenson DJ (2000) Influence of early plate tectonics on the thermal evolution and magnetic field of Mars. J Geophys Res 105:11969-11979

Olson P, Christensen U. R (2006) Dipole moment scaling for convection-driven planetary dynamos. Earth Planet Sci Lett 250:561-571

O'Neill C, Jellinek AM, Lenardic A (2007) Conditions for the onset of plate tectonics on terrestrial planets and moons. Earth Planet Sci Lett 261:20-32

Pommier A, Gaillard F, Malki M, Pichavant M (2010) Methodological re-evaluation of the electrical conductivity of silicate melts. Am Mineral 95:284-291

Reese CC, Solomatov VS (2010) Early martian dynamo generation due to giant impacts. Icarus 207:82-97

Rivoldini A, Hoolst TV, Verhoeven O, Mocquet A, Dehant V (2011) Geodesy constraints on the interior structure and composition of Mars. Icarus 213:451-472

Sohl F, Spohn T (1997) The interior structure of Mars: implications from SNC meteorites. J Geophys Res 102:1613-1635

Solomatov V (2007) Magma oceans and primordial mantle differentiation. In: Schubert G (ed). Treatise on Geophysics (2nd Ed.) Vol. 9. pp 81-104

Starchenko SV, Jones CA (2002) Typical velocities and magnetic field strengths in planetary interiors. Icarus 157:426-435

Stegman DR, Jellinek AM, Zatman SA, Baumgardner JR, Richards MA (2003) An early lunar dynamo driven by thermochemical mantle convection. Nature 421:143-146

Stevenson D (1983) Planetary magnetic fields. Rep Prog Phys 46:555-620

Stevenson D (2001) Mars' core and magnetism. Nature 412:214-219

Stevenson D (2003) Planetary magnetic fields. Earth Planet Sci Lett 208:1-11

Takahashi F, Matsushima M (2006) Dipolar and non-dipolar dynamos in a thin shell geometry with implications for the magnetic field of Mercury. Geophys Res Lett 33:10-10292006025792

Taylor J, Teanby NA, Wookey J (2013) Estimates of seismic activity in the Cerberus Fossae region of Mars. J Geophys Res 118:2570-2581

Weiss BP, Vali H, Baudenbacher FJ, Kirschvink JL, Stewart ST, Shuster DL (2002) Records of an ancient Martian magnetic field in ALH84001. Earth Planet Sci Lett 201:449-463

Whaler KA, Purucker ME (2005) A spatially continuous magnetization model for Mars. J Geophys Res 110:10-10292004002393 
Williams J-P, Nimmo F (2004) Thermal evolution of the Martian core: implications for an early dynamo. Geology 23:97-100

Wu C-C, Roberts PH (2009) On a dynamo driven by topographic precession. Geophys Astrophys Fluid Dyn 103:467-501

Ziegler LB, Stegman DR (2013) Implications of a long-lived basal magma ocean in generating Earth's ancient magnetic field. Geochem Geophys Geosyst 14:4735-4742

\section{Submit your manuscript to a SpringerOpen ${ }^{\circ}$ journal and benefit from:}

- Convenient online submission

- Rigorous peer review

- Open access: articles freely available online

- High visibility within the field

- Retaining the copyright to your article

Submit your next manuscript at $\gg$ springeropen.com 